\title{
Ex vivo vs. in vivo antibacterial activity of two antiseptics on oral biofilm
}

\begin{abstract}
Isabel Prada-López, Víctor Quintas, Maria A. Casares-De-Cal, Juan A. Suárez-Quintanilla, David Suárez-Quintanilla and Inmaculada Tomás *

Oral Sciences Research Group, Special Needs Unit, School of Medicine and Dentistry, University of Santiago de Compostela, Santiago de Compostela, Spain
\end{abstract}

Aim: To compare the immediate antibacterial effect of two application methods (passive immersion and active mouthwash) of two antiseptic solutions on the in situ oral biofilm.

OPEN ACCESS

Edited by:

Yuji Morita,

Aichi Gakuin University, Japan

Reviewed by:

Jean-Christophe Farges,

University Lyon 1, France

Fabian Cieplik,

University Medical Center

Regensburg, Germany

${ }^{*}$ Correspondence:

Inmaculada Tomás,

School of Medicine and Dentistry. Santiago de Compostela University,

C./ Entrerrios s/n, 15872,

Santiago de Compostela, Spain inmaculada.tomas@usc

Specialty section:

This article was submitted to Antimicrobials, Resistance and

Chemotherapy,

a section of the journa

Frontiers in Microbiology

Received: 02 May 2015 Accepted: 15 June 2015

Published: 02 July 2015

Citation

Prada-López I, Quintas V, Casares-De-Cal MA

Suárez-Quintanilla JA,

Suárez-Quintanilla D and Tomás I (2015) Ex vivo vs. in vivo antibacterial activity of two antiseptics on oral biofilm. Front. Microbiol. 6:655 doi: 10.3389/fmicb.2015.00655
Material and Methods: A randomized observer-masked crossover study was conducted. Fifteen healthy volunteers wore a specific intraoral device for $48 \mathrm{~h}$ to form a biofilm in three glass disks. One of these disks was used as a baseline; another one was immersed in a solution of $0.2 \%$ Chlorhexidine $(0.2 \% \mathrm{CHX})$, remaining the third in the device, placed in the oral cavity, during the $0.2 \% \mathrm{CHX}$ mouthwash application. After a 2-weeks washout period, the protocol was repeated using a solution of Essential Oils (EO). Samples were analyzed for bacterial viability with the confocal laser scanning microscope after previous staining with LIVE/DEAD ${ }^{\circledR}$ BacLight $^{\mathrm{TM}}$.

Results: The EO showed a better antibacterial effect compared to the $0.2 \% \mathrm{CHX}$ after the mouthwash application (\% of bacterial viability $=1.16 \pm 1.00 \%$ vs. $5.08 \pm 5.79 \%$, respectively), and was more effective in all layers $(p<0.05)$. In the immersion, both antiseptics were significantly less effective (\% of bacterial viability $=26.93 \pm 13.11 \%$, EO vs. $15.17 \pm 6.14 \%, 0.2 \% \mathrm{CHX}$; in the case of EO immersion, there were no significant changes in the bacterial viability of the deepest layer in comparison with the baseline.

Conclusions: The method of application conditioned the antibacterial activity of the $0.2 \% \mathrm{CHX}$ and $\mathrm{EO}$ solutions on the in situ oral biofilm. The in vivo active mouthwash was more effective than the ex vivo passive immersion in both antiseptic solutions. There was more penetration of the antiseptic inside the biofilm with an active mouthwash, especially with the EO. Trial registered in clinicaltrials.gov with the number NCT02267239. URL: https://clinicaltrials.gov/ct2/show/NCT02267239.

Keywords: antiseptic, chlorhexidine, essential oils, immersion, mouthwash, PL-biofilm

\section{Introduction}

The use of oral antiseptics is a recommended procedure for the chemical control of the oral biofilms. These oral antiseptics may kill the microorganisms, reduce bacterial virulence, and retard the dental plaque formation. Due to this action on the bacteria forming dental biofilms, a reduction of the oral disease is expected after their application (Corbin et al., 2011). 
Among dental practitioners, the most commonly prescribed oral antiseptics have been those including in their formulation Chlorhexidine (CHX) (Varoni et al., 2012) or Essential Oils (EO) (Axelsson, 2004). Although the antimicrobial effectiveness of both has been shown in previous studies (Gunsolley, 2010; Quintas et al., 2015), they exhibit different traits when certain methodologies are followed, which, in some cases, may limit the reliability of the results. Some of these differences have been recognized by the scientific community, who cautiously interpret the results of the studies that have used an in vitro-formed biofilm (Auschill et al., 2004). The use of determinate bacteria to create a biofilm in an artificial environment may result in a measure of antiseptic effectiveness that may not be representative of the in situ situation (Auschill et al., 2005).

Bacteria living in dental plaque develop relationships forming an extracellular matrix which is a highly resistant threedimensional (3-D) structure. This association makes the biofilm bacteria from 10 to 1000 times more resistant to an antiseptic (Fine et al., 2001; Davies, 2003). Given this, the handling of the in situ formed biofilm is an important characteristic that studies involving oral biofilms should take into account. In some studies the 3-D structure of the oral biofilm is altered when the sample is collected (Pan et al., 2000; Fine et al., 2005) or during the analysis process (Jentsch et al., 2002; Vitkov et al., 2005). The distortion of the 3-D structure probably influences the quantification of the antiseptic effectiveness. To avoid this, specific oral devices have been designed to allow the formation of a non-disturbed biofilm, similar to the dental plaque, which has been called plaque-like biofilm (PL-biofilm) (Prada-López et al., 2015a; Quintas et al., 2015). Furthermore, the use of confocal laser scanning microscope (CLSM) has allowed the possibility to analyse the in situ biofilm in their natural hydrated state without losing its complex structure (Arweiler et al., 2004). The CLSM has been used in combination with dual live/dead staining solutions. This has given to investigators the possibility to analyse the viability of a non-disturbed in situ oral biofilm, before and after the application of the antiseptics. Among the different available staining solutions, the SYTO 9 and propidium iodide has been one of the most successfully employed combinations of fluorochromes for visualizing dental plaque (Fuchslocher Hellemann et al., 2013; Hannig et al., 2013; Tawakoli et al., 2013; Prada-López et al., 2015a; Quintas et al., 2015).

Recently, some authors have stated that the methodology of the application of a given antiseptic could be an important factor which might condition the results on oral antiseptic effectiveness (Quintas et al., 2015). Commonly, in studies with undisturbed biofilm, the application of the antiseptic has been an ex vivo immersion of the sample into the solution (Zaura-Arite et al., 2001; Dong et al., 2010; Gosau et al., 2010; von Ohle et al., 2010). However, in recent series about the bacterial effect of CHX and $\mathrm{EO}$, the participants have undergone an active mouthwash with

\footnotetext{
Abbreviations: $0.2 \%$ CHX, solution of $0.2 \%$ of Chlorhexidine; EO, Essential Oils; PL-biofilm, plaque-like biofilm; IDODS, intraoral device of overlaid disk-holding splints; Im- $0.2 \% \mathrm{CHX}$, immersion in $0.2 \%$ Chlorhexidine; Im-EO, immersion in Essential Oils; Mw-0.2\%CHX, mouthwash with $0.2 \%$ Chlorhexidine; Mw-EO, mouthwash with Essential Oils.
}

the antiseptic in vivo (García-Caballero et al., 2013; Quintas et al., 2015), differing from the previously described results, mainly in regard to the EO activity.

Based on these previous findings, the authors of the present study intended to assess if the methodology of the antiseptic application might condition the obtained results in terms of bacterial viability of the PL-biofilm. Therefore, the objective of the present study was to compare the immediate effect of two antiseptic application methods, using separate solutions of $0.2 \%$ $\mathrm{CHX}$ and EO applied either by a passive immersion or an active mouthwash (ex vivo vs. in vivo exposure).

\section{Material and Methods}

The present study was designed as a randomized, observermasked, crossover study. The immediate effect of $0.2 \% \mathrm{CHX}$ and EO solutions was tested using them separately in immersion and mouthwash application on an in situ model of PL-biofilm growth. The supporting CONSORT checklist is available as supporting information (supplementary Table 1). This project got the approval of the Clinical Research Ethics Committee of Galicia (number 2012/393) and registered in clinicaltrials.gov with the number NCT02267239. URL: https://clinicaltrials.gov/ ct2/show/NCT02267239.

\section{Selection of the Study Group}

To calculate an a priori sample size, the following statistical criteria were established: an effect size of 0.35 , an alpha error of 0.05 and a statistical power of $87 \%$. Assuming these criteria and using the repeated measures ANOVA test, a sample size of 15 subjects was required. The sample size calculation was performed using the program $G^{\star}$ Power 3.1.5. The participants were recruited among dental students at the Faculty of Medicine and Dentistry of Santiago de Compostela (Spain), where volunteer enrolment was asked by responding to advertisements for the participation in a research study at the faculty hall. All of these volunteers were revised by the same trained clinician to ensure they fulfilled all inclusion and exclusion criteria. The volunteers chosen met the same inclusion and exclusion criteria of previous publications of our group (García-Caballero et al., 2013; PradaLópez et al., 2015a; Quintas et al., 2015). The inclusion criteria were the following: being systemically healthy adult volunteers between 20 and 45 years old, who presented a good oral health status: a minimum of 24 permanent teeth with no evidence of gingivitis or periodontitis (Community Periodontal Index score $=0)(\mathrm{WHO}, 1997)$ and an absence of untreated caries at the beginning of the study. The following exclusion criteria were applied: smoker or former smoker, presence of dental prostheses or orthodontic devices, antibiotic treatment or routine use of oral antiseptics in the previous 3 months, and presence of any systemic disease that could alter the production or composition of saliva. Before the start of each phase, a full mouth scaling with ultrasonic instruments and teeth polishing with rubber cup after dental disclosure was performed by the same trained clinician on all selected participants (Figure 1). Written informed consent was obtained from all participants in the study. 


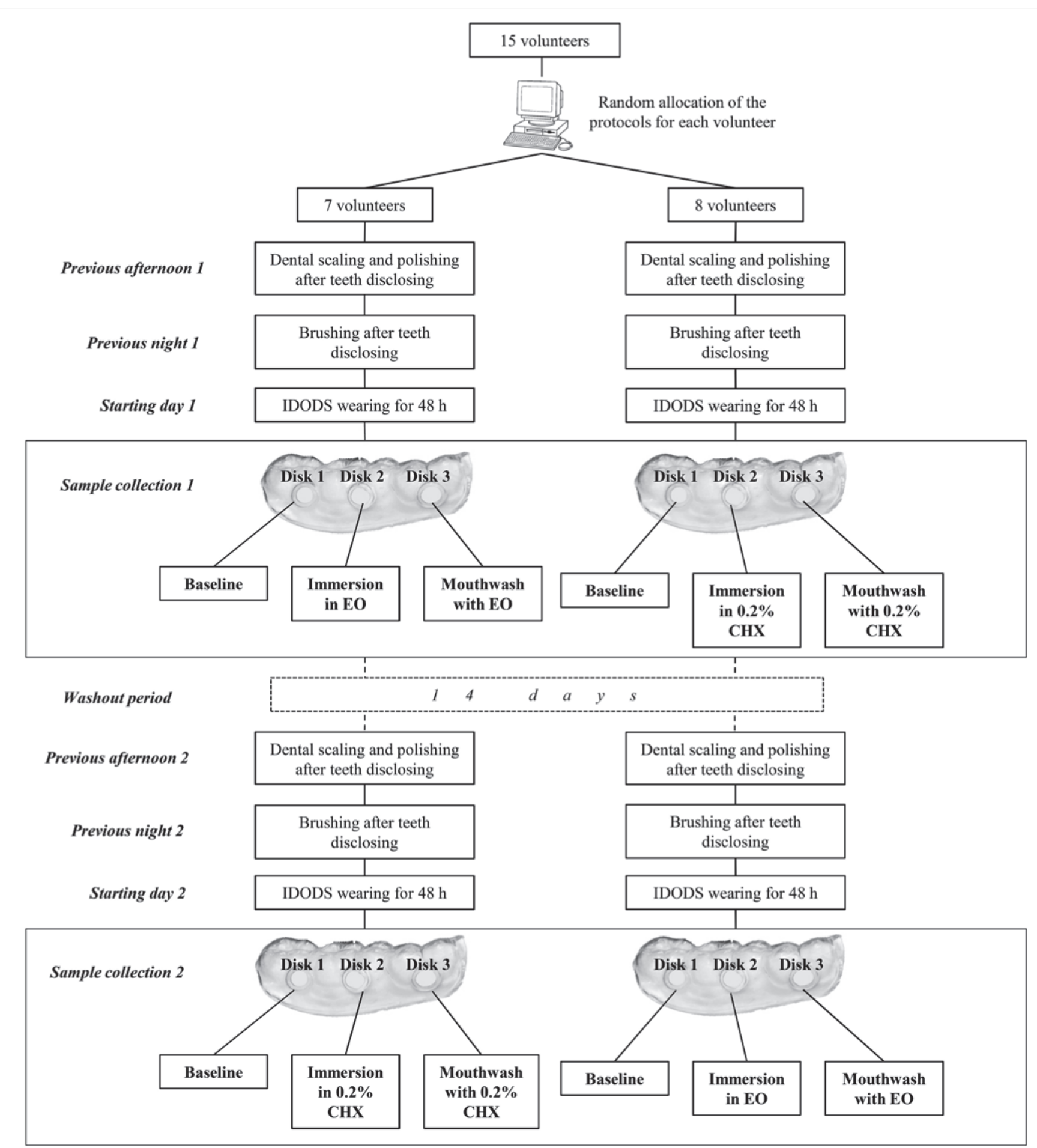

FIGURE 1 | Protocol of the study.

Intraoral Device of Overlaid Disk-Holding Splints (IDODS) for Biofilm in Situ Formation

Some in situ models for the growth of the biofilm have been previously described (Netuschil et al., 1998; Auschill et al.,
2001, 2005; Arweiler et al., 2004). After their consideration, an individualized splint of a lower hemi-arch was created for each volunteer, following the same protocol given in previous studies (García-Caballero et al., 2013; Prada-López et al., 2015a,b; 
Quintas et al., 2015). The Intraoral Device of Overlaid Diskholding Splints (IDODS) worn by the volunteers held three glass disks (6 $\mathrm{mm}$ in diameter, $1 \mathrm{~mm}$ thickness); these were polished at 800 grit. This splint has been already described in previous own works (Prada-López et al., 2015a,b; Quintas et al., 2015) (Figure 2).

The IDODS with the glass disks was worn by the subjects for $48 \mathrm{~h}$ (2 days) to favor growth of the PL-biofilm. They were allowed to withdraw it from the oral cavity only during meals and to perform oral hygiene measures (while it had to be stored in a previously provided opaque container in humid conditions). In order to not to disturb the growing of the PLbiofilm, volunteers could not use any toothpaste or mouthwash as a complement for the mechanical removal of bacterial plaque.

\section{Application of the $0.2 \% \mathrm{CHX}$ and EO to PL-Biofilm}

The sample analysis was divided into two phases, each of following the application protocol of the antiseptic. The first consisted of withdrawing the glass disks one by one from the splint (Figure 1) after the volunteer had worn it for $48 \mathrm{~h}$. The distal of these disks was used as a baseline sample. The second disk underwent one of two protocols:

A single, $30-\mathrm{s}$ immersion in $1 \mathrm{~mL}$ of $0.2 \%$ Chlorhexidine (Oraldine Perio ${ }^{\circledR}$, Johnson \& Johnson, Madrid, Spain) (Im- $0.2 \%$ CHX).

-OR-

A single, 30-s immersion in $1 \mathrm{~mL}$ of Essential Oils in a hydroalcoholic solution (Listerine Mentol, Listerine ${ }^{\circledR}$, Johnson \& Johnson, Madrid, Spain) (Im-EO).

Next, the second phase of the study was conducted. The last disk in the splint, placed in the oral cavity, was withdrawn after the volunteer performed the following under supervision:

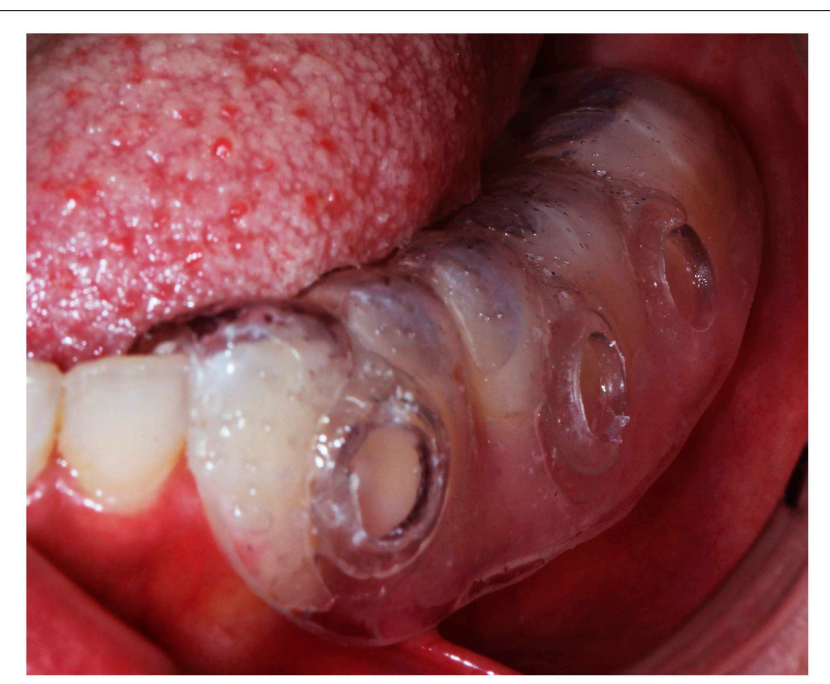

FIGURE 2 | Intraoral view of the Intraoral Device Overlaid Disk-holding Splint (IDODS).
A single, 30-s mouthwash with $10 \mathrm{~mL}$ of $0.2 \%$ Chlorhexidine (Oraldine Perio ${ }^{\circledR}$, Johnson \& Johnson, Madrid, Spain) (Mw-0.2\% CHX), following the instructions of the manufacturer. -OR-

A single, 30-s mouthwash with $20 \mathrm{~mL}$ of Essential Oils in a hydroalcoholic solution (Listerine Mentol, Listerine ${ }^{\circledR}$, Johnson \& Johnson, Madrid, Spain) (Mw-EO), following the instructions of the manufacturer.

Using an internet-based balanced randomization system (www.randomization.com) that indicated the antiseptic each subject would use first and second, as well as the hemi-arch (left or right) selected for the immersion and mouthwash. All subjects performed the two tests with a rest period of 14 days in-between (Figure 1).

\section{Collection of the Samples of PL-Biofilm}

On the day of the experiment, the volunteers were not allowed to eat or drink during the course of the tests. PL-biofilm samples collection was done individually (samples were taken from just one volunteer per day), starting at 8.30 AM (first baseline sample and immersions) and finished at 9.30 AM (mouthwash).

Immediately after the glass disks were withdrawn from the splints, they were submerged in $100 \mu \mathrm{L}$ of fluorescence solution LIVE/DEAD ${ }^{\circledR}$ BacLight ${ }^{\mathrm{TM}}$ and kept in a dark chamber at room temperature for $15 \mathrm{~min}$. A single investigator, masked to the study design, performed the microscopic observation using a Leica TCS SP2 laser scanning spectral confocal microscope (Leica Microsystems Heidelberg GmbH, Mannheim, Germany) with an HCX APOL 63x/0.9 water-immersion lens.

\section{Processing of the PL-Biofilm Samples}

In the present series, the same protocol described by Quintas et al. (2015) was followed to evaluate the different fields within the disks. Four selected fields (considered as representative of the whole sample), which were in the central part of each disk were evaluated; their mean measures of the thickness and bacterial viability represented the whole sample thickness and bacterial viability, respectively. The maximum biofilm thickness of each field was divided into three equivalent zones or same sized layers: outer layer (layer 1), middle layer (layer 2) and inner layer (layer 3).

The capture of the data was done with the same settings in all cases, according to previously presented parameters (Quintas et al., 2015) (Figure 3).

Quantification of bacterial viability was also done as previously presented (Quintas et al., 2015) using the cytofluorographic analysis (Leica Confocal Software) of XY images. In this analysis, the images of each fluorochrome were defined as "channels" (SYTO 9 occupies the green channel and propidium iodide the red channel). Square capture masks were used to measure the area occupied $\left(\mu \mathrm{m}^{2}\right)$ by the pixels in each channel, determining the total area occupied by the biofilm and the corresponding percentage of viability. The intensity range was considered a positive signal if it was between 100 and 255 . Determination of the mean percentage of bacterial viability in each field required sections with a minimum area of biofilm of $250 \mu \mathrm{m}^{2}$; the mean percentage of bacterial viability of the biofilm 


\section{PL-biofim maximum projection images of the $0.2 \% \mathrm{CHX}$ and $\mathrm{EO}$ series}

Baseline
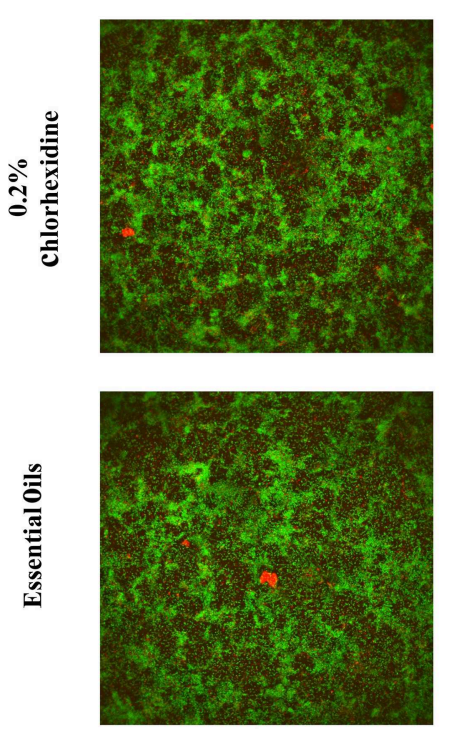

After Immersion
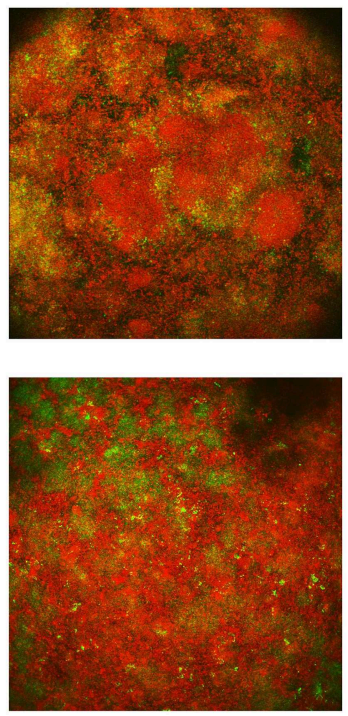

After Mouthwash
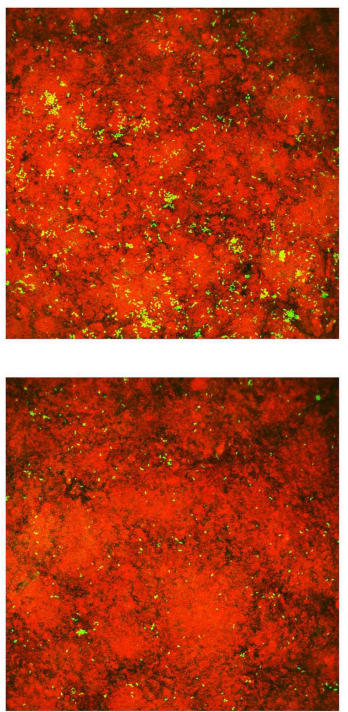

FIGURE 3 | Representative images of the PL-biofilm ("stacked projection" of images in the " $Z$ " axis) bacterial viability under basal conditions, after immersion and after mouthwash with $0.2 \%$ Chlorhexidine and Essential Oils. (They are images of representative fields of the PL-biofilm. It is a maximum projection of all obtained images in the plane $X Y$ in the $Z$ axis for a same field. That is commonly called "stacked projection." These images do not represent nor the outer, the middle or the inner layers, they represent all of them projected in the same axis). was calculated for the corresponding sample and for each biofilm layer.

\section{Statistical Analysis}

The data on thickness and bacterial viability in the PL-biofilm, were expressed as mean and standard deviation of the mean. The type of distribution of the quantitative variables analyzed was determined using the Kolmogorov-Smirnov test, obtaining a normal distribution for all values. Repeated measures ANOVA test and pairwise comparisons (with the Bonferroni correction) were used for the analysis of intra- and inter-application results for $0.2 \% \mathrm{CHX}$ and $\mathrm{EO}$ and inter-antiseptic solution results (including differentiating between the 3 biofilm layers). Measurements were statistical significant if the $p$ value less than 0.05 . The statistical analysis was performed by the PASW Statistics Base 20 package for Windows (IBM, Madrid, Spain).

\section{Results}

\section{Influence of the Application of Methods of $0.2 \%$ $\mathrm{CHX}$ and EO on the PL-Biofilm Thickness}

The thicknesses obtained in both baseline disks were 19.17 and $20.33 \mu \mathrm{m}$, before applying either $0.2 \%$ CHX or EO, respectively. After the Im-0.2\% CHX, the thickness was $17.64 \mu \mathrm{m}$ and $15.77 \mu \mathrm{m}$ after the mouthwash. When the applied antiseptic was the EO, the obtained thicknesses were 17.97 and $20.82 \mu \mathrm{m}$, after immersion and mouthwash, respectively. No significant differences were found in either case.

\section{Influence of the Application Methods of $0.2 \%$ $\mathrm{CHX}$ and EO on the PL-Biofilm Bacterial Viability}

The bacterial viability in the baseline disks was not significantly different between the two series of $0.2 \% \mathrm{CHX}$ and the $\mathrm{EO}$ $(72.21 \pm 10.48 \%$ vs. $75.72 \pm 14.33 \%)$.

After the Im- $0.2 \%$ CHX, the bacterial viability was significantly reduced to $15.17 \pm 6.14 \%$. In contrast, the bacterial viability after the Mw- $0.2 \% \mathrm{CHX}$ was $5.08 \pm 5.79 \%$ (Figure 4), which was significantly lower than the Im- $0.2 \% \mathrm{CHX}$ $(p=0.001)$. In addition, both results differed significantly from their baseline values $(p<0.001)$ (Table 1).

In the same way, the Im-EO significantly reduced the bacterial viability to $26.93 \pm 13.11 \%$. However, after Mw-EO, the bacterial viability was reduced to $1.16 \pm 1.00 \%$ (Figure 5), which was significantly lower than the Im-EO $(p<0.001)$. Besides, both results differed significantly from their baseline values $(p<$ 0.001) (Table 1).

In the comparison of the two antiseptics, the Im- $0.2 \% \mathrm{CHX}$ obtained significantly lower values of bacterial viability compared with the Im-EO $(15.17 \pm 6.14 \% \mathrm{CHX}$ vs. $26.93 \pm 13.11 \% \mathrm{EO}$, $p<0.05)$. On the other hand, the Mw-EO achieved significantly lower bacterial viability in comparison with the $\mathrm{Mw}-0.2 \% \mathrm{CHX}$ $(1.16 \pm 1.00 \%$ vs. $5.08 \pm 5.79 \%, p<0.05)$ (Table 2 ).

\section{Influence of the Application Methods of $\mathrm{CHX}$ and EO on the PL-Biofilm Bacterial Viability by Layers}

When accounting for the different layers, the values for baseline bacterial viability decreased progressively for deeper layers 


\title{
Total bacterial vitality and by layers in the $0.2 \%$ CHX series
}

\author{
CLSM sectional images \\ Mean bacterial vitality \% \\ (TOTAL and by layers)
}
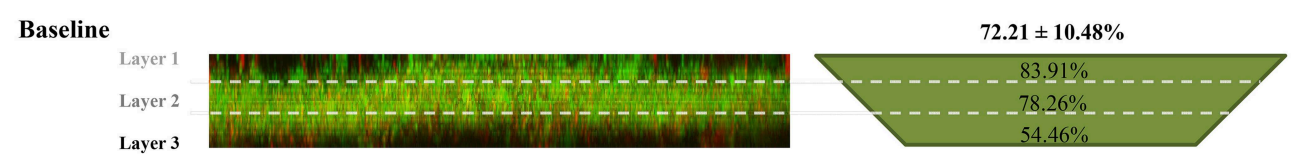

After Immersion

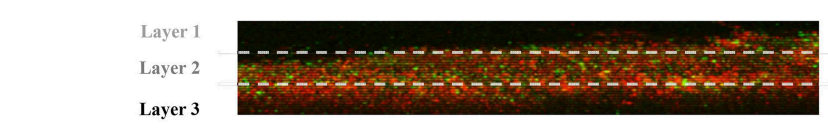

$15.17 \pm 6.14 \%$

After Mouthwash
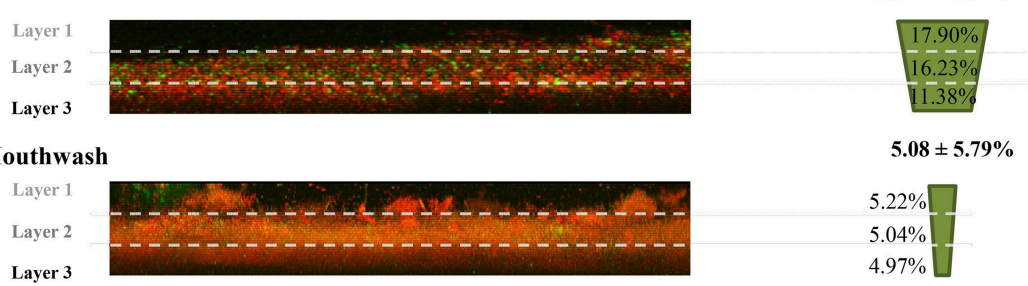

FIGURE 4 | Total bacterial viability and by PL-biofilm layers in the $\mathbf{0 . 2} \%$ Chlorhexidine series. (PL-biofilm, plaque like-biofilm; $0.2 \%$ CHX, $0.2 \%$ of Chlorhexidine; CLSM, confocal laser scanning microscope).

TABLE 1 | Inter-application analysis for $0.2 \%$ of Chlorhexidine and Essential Oils by layers.

\begin{tabular}{|c|c|c|}
\hline $\begin{array}{l}\text { Application method } \\
\text { (inter-application) }\end{array}$ & $0.2 \%$ Chlorhexidine & $\begin{array}{c}\text { Essential } \\
\text { Oils }\end{array}$ \\
\hline \multicolumn{3}{|l|}{ TOTAL } \\
\hline Baseline vs. immersion & $p<0.001$ & $p<0.001$ \\
\hline Baseline vs. mouthwash & $p<0.001$ & $p<0.001$ \\
\hline Immersion vs. mouthwash & $p=0.001$ & $p<0.001$ \\
\hline \multicolumn{3}{|l|}{ LAYER 1} \\
\hline Baseline vs. immersion & $p<0.001$ & $p<0.001$ \\
\hline Baseline vs. mouthwash & $p<0.001$ & $p<0.001$ \\
\hline Immersion vs. mouthwash & $p=0.001$ & $p=0.002$ \\
\hline \multicolumn{3}{|l|}{ LAYER 2} \\
\hline Baseline vs. immersion & $p<0.001$ & $p<0.001$ \\
\hline Baseline vs. mouthwash & $p<0.001$ & $p<0.001$ \\
\hline Immersion vs. mouthwash & $p=0.001$ & $p<0.001$ \\
\hline \multicolumn{3}{|l|}{ LAYER 3} \\
\hline Baseline vs. immersion & $p<0.001$ & - \\
\hline Baseline vs. mouthwash & $p<0.001$ & $p<0.001$ \\
\hline Immersion vs. mouthwash & $p=0.046$ & $p<0.001$ \\
\hline
\end{tabular}

(baseline sample prior to $0.2 \% \mathrm{CHX}$ application in layer $1=$ $83.91 \pm 9.51 \%$, layer $2=78.26 \pm 9.93 \%$, and layer $3=54.46 \pm$ 25.43\% (Figure 4 and Table 3); baseline sample prior to EO application in layer $1=89.53 \pm 8.34 \%$, layer $2=82.12 \pm 13.26 \%$, and layer $3=55.53 \pm 28.19 \%$ ) (Figure 5 and Table 3).

After the Im- $0.2 \%$ CHX protocol, the sample showed a homogeneous decrease in value from the baseline situation in all layers (Im-0.2\% CHX, layer $1=17.90 \pm 9.16 \%$, layer $2=16.23 \pm$ $7.14 \%$, and layer $3=11.38 \pm 5.81 \%, p<0.001$ in all cases). In addition, after the $\mathrm{Mw}-0.2 \% \mathrm{CHX}$ protocol, the bacterial viability was more reduced compared to the immersion protocol (Mw$0.2 \%$ CHX, layer $1=5.22 \pm 6.16 \%$, layer $2=5.04 \pm 6.40 \%$, layer
$3=4.97 \pm 5.00 \%, p<0.05$ comparing Im- $0.2 \% \mathrm{CHX}$ and its baseline) (Figure 4 and Table 1).

After the Im-EO protocol, the sample showed a general decrease from the baseline disk in the superficial layers (Im$\mathrm{EO}$, layer $1=16.18 \pm 12.38 \%$ and layer $2=25.60 \pm 14.51 \%$, $p<0.001$ in layers 1 and 2) but not in the deepest layer (Im$\mathrm{EO}$, layer $3=39.02 \pm 17.50 \%$ ). In contrast, the results after the Mw-EO showed a highly reduced bacterial viability in all layers of the PL-biofilm, thus it was significantly more effective at reducing bacterial viability than the immersion protocol (Mw-EO, layer 1 $=1.62 \pm 1.54 \%$, layer $2=1.12 \pm 1.16 \%$, and layer $3=0.72 \pm$ $0.56 \% ; p<0.05$ comparing Im-EO and its baseline) (Figure 5 and Table 1).

Comparing the two antiseptics, although both showed a significant reduction of bacterial viability after immersion in all layers, in layer 3 the $0.2 \%$ CHX solution showed more reduced bacterial viability than did the EO (layer 3 , Im- $0.2 \%$ CHX vs. Im$\mathrm{EO}, 11.38 \pm 5.81 \%$ vs. $39.02 \pm 17.50 \% ; p<0.001)$. In contrast, in comparison with the mouthwash application, the Mw-EO obtained lower bacterial viability than the $\mathrm{Mw}-0.2 \% \mathrm{CHX}$, in all layers $(p<0.05)$ (Table 2).

\section{Discussion}

To the best of the author's knowledge, there are no published studies which compare the immediate antibacterial effect of an oral antiseptic applied using the two methods referred to in much of the literature (ex vivo passive immersion and in vivo active mouthwash) within the same experiment (using the same volunteer and PL-biofilm within one growth period).

In the present series, glass disks were used instead of enamel ones for several reasons. The first, and more important, is that previous research (Netuschil et al., 1998) and own's (unpublished data) revealed that there were no significant differences in the bacterial viability and thickness of the 2-day PL-biofilm formed on enamel or polished glass analyzed with CLSM. This is a very 


\section{Total bacterial vitality and by layers in the EO series}

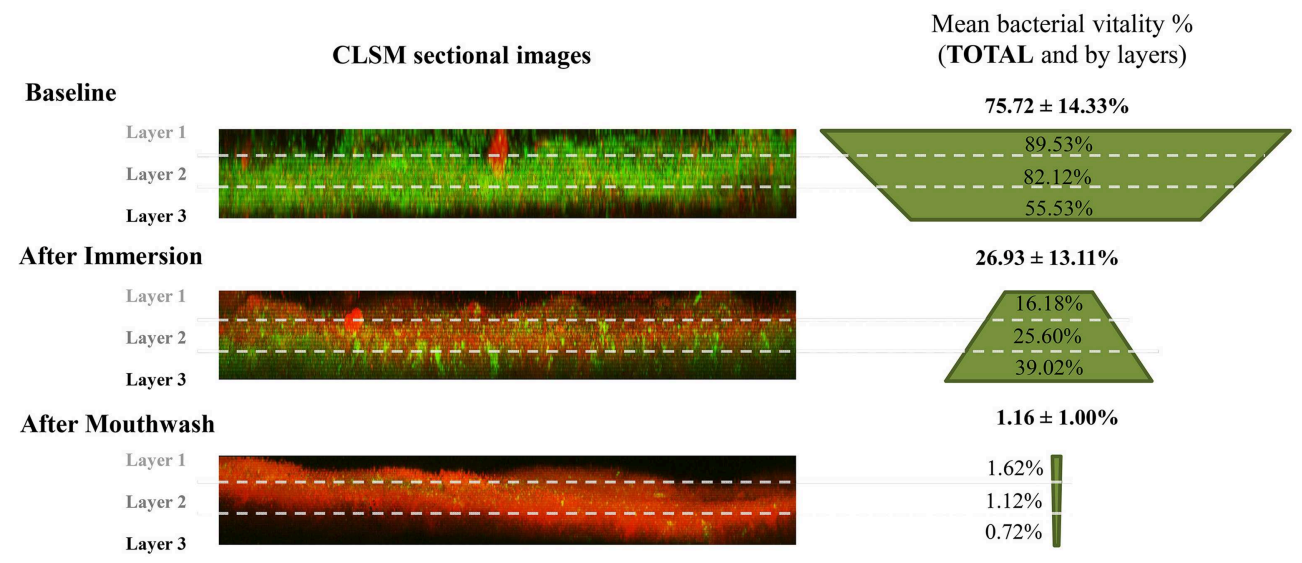

FIGURE 5 | Total bacterial viability and by PL-biofilm layers in the Essential Oils series. (PL-biofilm, plaque like-biofilm; EO, Essential Oils; CLSM, confocal laser scanning microscope).

TABLE 2 | Inter-antiseptic solution analysis between $0.2 \%$ of Chlorhexidine and Essential Oils by layers.

\begin{tabular}{lc}
\hline $\begin{array}{l}\text { Application method } \\
\text { (inter-antiseptic solution) }\end{array}$ & $\begin{array}{c}\mathbf{0 . 2 \%} \text { Chlorhexidine vs. } \\
\text { Essential Oils }\end{array}$ \\
\hline BASELINE & - \\
Total & - \\
Layer 1 & - \\
Layer 2 & - \\
Layer 3 & \\
IMMERSION & $p=0.007$ \\
Total & - \\
Layer 1 & - \\
Layer 2 & $p<0.001$ \\
Layer 3 & \\
MOUTHWASH & $p=0.020$ \\
Total & $p=0.041$ \\
Layer 1 & $p=0.034$ \\
Layer 2 & $p=0.006$ \\
Layer 3 &
\end{tabular}

important fact because it is much easier to obtain a regular glass disk in size and thickness than from enamel, being this crucial to the construction of the intraoral splints and the stability of the disks into it. Another reason is the difficulty of analysing the biofilm formed on an enamel disk due to the irregularity (it is not a plane surface) giving distorted images at the CLSM. In addition, although the sterility of the enamel disks could be achieved easily, there always could exist the latent risk of prion diseases, which would bring an important moral dilemma.

\section{PL-biofilm Thickness}

The present series showed a constant thickness of the PL-biofilm after all applied situations. The application of the antiseptic, either by immersion or mouthwash, did not change the basal
TABLE 3 | Intra-application analysis for $0.2 \%$ of Chlorhexidine and Essential Oils by layers.

\begin{tabular}{lcc}
\hline $\begin{array}{l}\text { Application method } \\
\text { (intra-application) }\end{array}$ & $\mathbf{0 . 2 \%}$ Chlorhexidine & $\begin{array}{c}\text { Essential } \\
\text { Oils }\end{array}$ \\
\hline BASELINE & $p=0.038$ & $p=0.021$ \\
Layer 1 vs. Layer 2 & $p=0.005$ & $p=0.001$ \\
Layer 1 vs. Layer 3 & $p=0.006$ & $p=0.001$ \\
Layer 2 vs. Layer 3 & - & $p=0.004$ \\
IMMERSION & - & $p<0.001$ \\
Layer 1 vs. Layer 2 & - & $p<0.001$ \\
Layer 1 vs. Layer 3 & - & $p=0.021$ \\
Layer 2 vs. Layer 3 & - & - \\
MOUTHWASH & - & - \\
Layer 1 vs. Layer 2 & - & \\
Layer 1 vs. Layer 3 & - & \\
Layer 2 vs. Layer 3 & - & \\
\hline
\end{tabular}

thickness. The previous literature support this situation, no matter the antiseptic solution or the methodology of application used in the studies (Zaura-Arite et al., 2001; Dong et al., 2010; von Ohle et al., 2010; García-Caballero et al., 2013; Quintas et al., 2015), so that a single application of $0.2 \%$ CHX or EO does not affect the thickness of a mature biofilm.

\section{PL-biofilm Bacterial Viability}

In the literature, the mean bacterial viability in a 2-day PL-biofilm oscillated between 60 and 77\% (von Ohle et al., 2010; Gu et al., 2012; García-Caballero et al., 2013; Prada-López et al., 2015a; Quintas et al., 2015), the present series showed results in this range (72 and $76 \%$ in both baseline situations, before $\mathrm{CHX}$ and EO applications, respectively).

The mean bacterial viability after an Im- $0.2 \%$ CHX ranged from 0.7 to $35.16 \%$ in the previous scientific literature (ZauraArite et al., 2001; Gosau et al., 2010; von Ohle et al., 2010). Such 
wide variations might be caused by the different methodologies used in the studies. These differences mainly arise because of a range of factors, such as the antiseptic concentration or the time lapse of application. In their study on PL-biofilm, von Ohle et al. (2010) chose a $0.1 \%$ CHX concentration and their immersion times varied between 1 and $10 \mathrm{~min}$. This protocol contrasts with that followed by Zaura-Arite et al. (2001) and Gosau et al. (2010), who evaluated a commercial 0.2\% CHX concentration, and selected an immersion time lapse of $1 \mathrm{~min}$. Despite the obvious methodological differences with other series (time of exposure and concentration), the results of the present study in terms of bacterial viability of the PL-biofilm after an Im- $0.2 \%$ CHX (approximately a 15\%) are in accordance with the results reported in the previous literature (Zaura-Arite et al., 2001; Gosau et al., 2010; von Ohle et al., 2010). Respect to the applied CHX protocol, the manufacturer recommendations in terms of time of application were followed (30 s).

Regarding the Mw- $0.2 \% \mathrm{CHX}$, there are few evaluations of the bacterial viability of the PL-biofilm after an active mouthwash with this antiseptic (García-Caballero et al., 2013; Quintas et al., 2015). In the present series, the bacterial viability was near to $5 \%$ which is consistent with that reported in previous studies (García-Caballero et al., 2013; Quintas et al., 2015).

Concerning the mean bacterial viability after an Im-EO, the results found in the literature ranged from 23 to $31 \%$ (Dong et al., 2010; Gosau et al., 2010). In these cases, the antiseptic concentration did not vary from one study to another. This is probably one of the reasons why the range is narrower for the $\mathrm{EO}$ than for the 0.2\% CHX. Gosau et al. (2010) and Dong et al. (2010) immersed a 12 and 48 h-PL-biofilm for $1 \mathrm{~min}$, respectively. The present series showed a mean bacterial viability within the named range (approximately a 27\%).

In regard to the assessment of the efficacy of an active Mw-EO, to the best of the author's knowledge, there is only one study in which the bacterial viability of the PL-biofilm has been evaluated (Quintas et al., 2015). This study showed a very low bacterial viability $30 \mathrm{~s}$ after the $\mathrm{Mw}-\mathrm{EO}$, near to $1 \%$, which is similar to the present results.

Despite the visible lack of previous literature, the results of the present series, in terms of bacterial viability, have a clear interpretation, according to the authors: doing an active mouthwash greatly reduces the bacterial viability of the PLbiofilm, more so than doing an immersion with the same antiseptic. When differentiating between $0.2 \% \mathrm{CHX}$ and EO, we found doing an Im- $0.2 \%$ CHX was more effective than doing an Im-EO.

On the other hand, when a mouthwash was done, the EO solution was more effective than the $0.2 \%$ CHX. In previous research of the authors (Quintas et al., 2015), the immediate effect of the EO vs. $0.2 \% \mathrm{CHX}$ has been already presented and discussed in detail.

\section{PL-biofilm Bacterial Viability by Layers}

The distribution of the bacterial viability into the PL-biofilm in the baseline disks was significantly lower in the deepest layers. This distribution pattern of viability, in which vital bacteria overlay non-vital bacteria, has been previously described in other in vivo biofilm studies that analyzed bacterial viability in layers (Arweiler et al., 2004; García-Caballero et al., 2013; Prada-López et al., 2015a; Quintas et al., 2015).

After the Im- $0.2 \% \mathrm{CHX}$, the bacterial viability decreased significantly in all layers. However, there were no differences among the layers (18\% outer, $16 \%$ middle and $11 \%$ inner layer). Zaura-Arite et al. (2001) analyzed the bacterial viability in the different layers after a 1 min immersion in $0.2 \%$ CHX showing its efficacy as well, but with wide ranges of values (outer layer $=16$ $42 \%$, middle layer $=19-55 \%$ and inner layer $=21-58 \%)$. These data were probably obtained due to the small sample size and the characteristics of the volunteers (three heavy plaque-formers and three light plaque-formers).

On the other hand, the Mw- $0.2 \% \mathrm{CHX}$ reduced the bacterial viability similarly in all layers, being these findings in accordance with those previously described in situ studies (García-Caballero et al., 2013; Quintas et al., 2015). This reduction in bacterial viability obtained by mouthwash, as recorded by layers, was higher than the obtained from the immersion method.

In the present series, when applying the EO antiseptic, the bacterial viability of the PL-biofilm was reduced in all layers after the immersion. The outer layer showed significant less viability than the other two (16\% in the outer layer vs. $26 \%$ in the middle and $39 \%$ in the deepest layer). In the same manner, Dong et al. (2010), after a 1-min Im-EO found less bacterial viability in the outer layer (outer layer $=22 \%$ vs. middle layer $=34 \%$, and inner layer $=37 \%$ ). In both studies, a reduction in the bacterial viability was shown, but a different spatial distribution in the bacterial viability compared to their baselines could be seen (Figure 5). While in the baseline sample the bacterial viability decreased from the outer to the inner layers, after an Im-EO, this distribution was inverted, showing an increase in the bacterial viability from the outer to the inner layers. This fact could be explained by the low capacity of the EO solution to penetrate mature biofilms, resulting in a loss of efficacy in the deepest layers of the PL-biofilm. In the contrast, this effect seen after the Im-EO was completely lost after the Mw-EO. The EO applied following the manufacturer's instructions (a single mouthwash with $20 \mathrm{~mL}$ for $30 \mathrm{~s}$ ) was clearly more effective in all layers than the simple immersion, achieving bacterial viability results near to $0 \%$ in the three layers.

The results of the present series confirmed that, to properly assess the immediate antibacterial effect of $0.2 \% \mathrm{CHX}$ and $\mathrm{EO}$, an in vivo active mouthwash following the manufacturer's recommendations should be done. This series has also shown that an active mouthwash helped to maximize the efficacy of the $0.2 \%$ CHX solution and, mainly, the EO solution compared to a single immersion. In addition, the findings of this investigation suggest cautious interpretation of the results of studies that followed an ex vivo antiseptic application (immersion) in PL-biofilm. This previous literature about antimicrobial activity of both $0.2 \% \mathrm{CHX}$ and $\mathrm{EO}$ relies on in vitro and in situ studies that do not follow proper methodologies, pretending to equate a simple immersion (ex vivo) with an active mouthwash (in vivo). To some extent, this equation could be considered valid when isolated bacteria are studied. However, when talking about bacteria associated in a more complex structure such as a naturally-formed biofilm 
(that is 10-1000 times more resistant than bacteria in planktonic phase) (Fine et al., 2001), something more than the simple contact with the antiseptic is needed. This higher activity of the antiseptic when applied as a mouthwash could be due to the hydrodynamic forces that appear in the mouth thank to the action of the tongue, cheeks and other muscles of the oral cavity that contribute to the movement of the mouthwash throughout all the surfaces of the mouth. This movement could achieve something that the passive immersion could not, which is breaking of the surface force of the PL-biofilm, being this crucial for the antiseptic penetration. This is the same theory followed in endodontics with the "hydrodynamic activation" of the antiseptic in the interior of the radicular canal by agitation (Weller et al., 1980; Peeters et al., 2014). This movement achieves to break the surface force of small root canals contributing to maximize the chemical action of the antiseptic (Peeters et al., 2014).

Finally, the authors would like to point out another possible differentiating variable which may condition the antiseptic effectiveness: the temperature. In the ex vivo experiment, the antiseptic was at room temperature (between 18 and $20^{\circ} \mathrm{C}$ ). However, when the application was in vivo, the temperature of the antiseptic solution rose by several degrees. In future investigations, it would be interesting to study the role that the temperature might play in the antimicrobial effect of the antiseptic.

\section{Conclusion}

The method of application conditioned the antibacterial activity of the $0.2 \%$ Chlorhexidine and the Essential Oils on the plaque-like biofilm. The in vivo active mouthwash protocol was more effective than the ex vivo passive immersion in both antiseptic solutions, conditioning the obtained results. There was more penetration of the antiseptic inside the biofilm with

\section{References}

Arweiler, N. B., Hellwig, E., Sculean, A., Hein, N., and Auschill, T. M. (2004). Individual vitality pattern of in situ dental biofilms at different locations in the oral cavity. Caries Res. 38, 442-447. doi: 10.1159/000079625

Auschill, T. M., Arweiler, N. B., Netuschil, L., Brecx, M., Reich, E., and Sculean, A. (2001). Spatial distribution of vital and dead microorganisms in dental biofilms. Arch. Oral. Biol. 46, 471-476. doi: 10.1016/S0003-9969(00) 00136-9

Auschill, T. M., Hein, N., Hellwig, E., Follo, M., Sculean, A., and Arweiler, N. B. (2005). Effect of two antimicrobial agents on early in situ biofilm formation. J. Clin. Periodontol. 32, 147-152. doi: 10.1111/j.1600-051X.2005. 00650.x

Auschill, T. M., Hellwig, E., Sculean, A., Hein, N., and Arweiler, N. B. (2004). Impact of the intraoral location on the rate of biofilm growth. Clin. Oral Investig. 8, 97-101. doi: 10.1007/s00784-004-0255-6

Axelsson, P. (2004). Preventive Materials, Methods and Programs, 1st Edn. Surrey: Quintessence.

Corbin, A., Pitts, B., Parker, A., and Stewart, P. S. (2011). Antimicrobial penetration and efficacy in an in vitro oral biofilm model. Antimicrob. Agents Chemother. 55, 3338-3344. doi: 10.1128/AAC.00206-11

Davies, D. (2003). Understanding biofilm resistance to antibacterial agents. Nat. Rev. Drug Discov. 2, 114-122. doi: 10.1038/nrd1008 an active mouthwash, especially when the Essential Oils were used.

According to the results of the present study, future investigations on oral antiseptics should take into account the methodology of the application. To obtain a situation as close as possible to the clinical reality, the plaque-like biofilm should be formed in vivo. In addition, the antiseptic application should be in situ, with an active mouthwash or, at least, take into consideration the role that the movement of the solution may have in the antiseptic antimicrobial activity.

\section{Author Contributions}

Conception and design the experiments: IT, JS, DS. Performed the experiments: IP, VQ. Analyzed data: IT, MACDC. Interpretation of the data: IP, VQ. Drafting and revising the manuscript: IP, VQ, MACDC, IT. Final approval: IT, JS, DS. Agreement: IP, VQ, MACDC, JS, DS, IT.

\section{Acknowledgments}

This work was supported by project PI11/01383 from Carlos III Institute of Health (General Division of Evaluation and Research Promotion, Madrid, Spain), which is integrated in National Plan of Research, Development and Innovation (PN I+D+I 2008-2011). This project was cofinanced by European Regional Development Fund (ERDF 2007-2013). The funders had no role in study design, data collection and analysis, decision to publish, or preparation of the manuscript.

\section{Supplementary Material}

The Supplementary Material for this article can be found online at: http://journal.frontiersin.org/article/10.3389/fmicb. 2015.00655
Dong, W. L., Zhou, Y. H., Li, C. Z., Liu, H., Shang, S. H., and Pan, B. Q. (2010). Establishment and application of an intact natural model of human dental plaque biofilm. Shanghai Kou Qiang Yi Xue 19, 196-201.

Fine, D. H., Furgang, D., and Barnett, M. L. (2001). Comparative antimicrobial activities of antiseptic mouthrinses against isogenic planktonic and biofilm forms of Actinobacillus actinomycetemcomitans. J. Clin. Periodontol. 28, 697-700. doi: 10.1034/j.1600-051x.2001.028007697.x

Fine, D. H., Furgang, D., Sinatra, K., Charles, C., McGuire, A., and Kumar, L. D. (2005). In vivo antimicrobial effectiveness of an essential oil-containing mouth rinse $12 \mathrm{~h}$ after a single use and 14 days' use. J. Clin. Periodontol. 32, 335-340. doi: 10.1111/j.1600-051x.2005.00674.x

Fuchslocher Hellemann, C., Grade, S., Heuer, W., Dittmer, M. P., Stiesch, M., Schwestka-Polly, R., et al. (2013). Three-dimensional analysis of initial biofilm formation on polytetrafluoroethylene in the oral cavity. J. Orofac. Orthop. 74, 458-467. doi: 10.1007/s00056-013-0174-8

García-Caballero, L., Quintas, V., Prada-López, I., Seoane, J., Donos, N., and Tomás, I. (2013). Chlorhexidine substantivity on salivary flora and plaque-like biofilm: an in situ model. PLoS ONE 8:e83522. doi: 10.1371/journal.pone.0083522

Gosau, M., Hahnel, S., Schwarz, F., Gerlach, T., Reichert, T. E., and Burgers, R. (2010). Effect of six different peri-implantitis disinfection methods on in vivo human oral biofilm. Clin. Oral Implants Res. 21, 866-872. doi: 10.1111/j.16000501.2009.01908.x 
Gu, H., Fan, D., Gao, J., Zou, W., Peng, Z., Zhao, Z., et al. (2012). Effect of $\mathrm{ZnCl}_{2}$ on plaque growth and biofilm vitality. Arch. Oral Biol. 57, 369-375. doi: 10.1016/j.archoralbio.2011.10.001

Gunsolley, J. C. (2010). Clinical efficacy of antimicrobial mouthrinses. J. Dent. 38(Suppl. 1), S6-S10. doi: 10.1016/S0300-5712(10)70004-X

Hannig, C., Kirsch, J., Al-Ahmad, A., Kensche, A., Hannig, M., and Kummerer, K. (2013). Do edible oils reduce bacterial colonization of enamel in situ? Clin. Oral Investig. 17, 649-658. doi: 10.1007/s00784-012-0734-0

Jentsch, H., Hombach, A., Beetke, E., and Jonas, L. (2002). Quantitative transmission electron microscopic study of dental plaque-an in vivo study with different mouthrinses. Ultrastruct. Pathol. 26, 309-313. doi: 10.1080/01913120290104584

Netuschil, L., Reich, E., Unteregger, G., Sculean, A., and Brecx, M. (1998). A pilot study of confocal laser scanning microscopy for the assessment of undisturbed dental plaque vitality and topography. Arch. Oral Biol. 43, 277-285. doi: 10.1016/S0003-9969(97)00121-0

Pan, P., Barnett, M. L., Coelho, J., Brogdon, C., and Finnegan, M. B. (2000). Determination of the in situ bactericidal activity of an essential oil mouthrinse using a vital stain method. J. Clin. Periodontol. 27, 256-261. doi: 10.1034/j.1600051x.2000.027004256.x

Peeters, H. H., Iskandar, B., Suardita, K., and Suharto, D. (2014). Visualization of the removal of trapped air from the apical region of the straight root canal models generating 2-phase intermittent counter flow during ultrasonically activated irrigation. J. Endod. 40, 857-861. doi: 10.1016/j.joen.2013.10.011

Prada-López, I., Quintas, V., Donos, N., Suarez-Quintanilla, D., and Tomás, I. (2015a). Characteristics of in situ oral biofilm after 2 and 4 days of evolution. Quintessence Int. 46, 287-298. doi: 10.3290/j.qi.a33402

Prada-López, I., Quintas, V., and Tomás, I. (2015b). The intraoral device of overlaid disk-holding splintsb as a new in situ oral biofilm model. J. Clin. Exp. Dent. 7, 126-132. doi: 10.4317/jced.52093

Quintas, V., Prada-López, I., Prados-Frutos, J. C., and Tomás, I. (2015). In situ antimicrobial activity on oral biofilm: essential oils vs. $0.2 \%$ chlorhexidine. Clin. Oral Investig. 19, 97-107. doi: 10.1007/s00784-014-1224-3
Tawakoli, P. N., Al-Ahmad, A., Hoth-Hannig, W., Hannig, M., and Hannig, C. (2013). Comparison of different live/dead stainings for detection and quantification of adherent microorganisms in the initial oral biofilm. Clin. Oral Investig. 17, 841-850. doi: 10.1007/s00784-012-0792-3

Varoni, E., Tarce, M., Lodi, G., and Carrassi, A. (2012). Chlorhexidine (CHX) in dentistry: state of the art. Minerva Stomatol. 61, 399-419.

Vitkov, L., Hermann, A., Krautgartner, W. D., Herrmann, M., Fuchs, K., Klappacher, M., et al. (2005). Chlorhexidine-induced ultrastructural alterations in oral biofilm. Microsc. Res. Techniq. 68, 85-89. doi: 10.1002/jemt. 20238

von Ohle, C., Gieseke, A., Nistico, L., Decker, E. M., DeBeer, D., and Stoodley, P. (2010). Real-time microsensor measurement of local metabolic activities in ex vivo dental biofilms exposed to sucrose and treated with chlorhexidine. Appl. Environ. Microbiol. 76, 2326-2334. doi: 10.1128/AEM.02090-09

Weller, R. N., Brady, J. M., and Bernier, W. E. (1980). Efficacy of ultrasonic cleaning. J. Endod. 6, 740-743. doi: 10.1016/S0099-2399(80)80185-3

WHO. (1997). "Oral health surveys, basic methods," in World Health Organization, 4th Edn., ed W. H. Van Palenstein Helderman (Geneva: WHO), 36-38.

Zaura-Arite, E., van Marle, J., and ten Cate, J. M. (2001). Conofocal microscopy study of undisturbed and chlorhexidine-treated dental biofilm. J. Dent. Res. 80, 1436-1440. doi: 10.1177/00220345010800051001

Conflict of Interest Statement: The authors declare that the research was conducted in the absence of any commercial or financial relationships that could be construed as a potential conflict of interest.

Copyright (c) 2015 Prada-López, Quintas, Casares-De-Cal, Suárez-Quintanilla, Suárez-Quintanilla and Tomás. This is an open-access article distributed under the terms of the Creative Commons Attribution License (CC BY). The use, distribution or reproduction in other forums is permitted, provided the original author(s) or licensor are credited and that the original publication in this journal is cited, in accordance with accepted academic practice. No use, distribution or reproduction is permitted which does not comply with these terms. 